\title{
Recurrence of primary spontaneous pneumothorax
}

\author{
R T Sadikot, T Greene, K Meadows, A G Arnold
}

\begin{abstract}
Background - Primary spontaneous pneumothorax (PSP) is a common clinical problem and its incidence is thought to be increasing. The risk of recurrence is high and various studies quote rates of $20-60 \%$. Factors which may or may not predispose to recurrence have not yet been established.

Methods - In a study period of four years 291 cases with a diagnosis of pneumothorax were reviewed; 153 patients with PSP were included in the study. Their risk of recurrence was analysed with particular reference to the following variables: age, sex, height and body mass index (BMI) of the patient, the initial size of pneumothorax, the smoking status of the patient, and the primary form of treatment employed. Univariate analysis was carried out by $\chi^{2}$ testing and multivariate analysis was calculated by a logistic regression model.
\end{abstract}

Results - A retrospective study of 275 episodes of PSP in 153 patients over a four year period confirmed a high incidence of recurrence $(54.2 \%)$. PSP was twice as common in men as in women, though women were significantly more likely to develop a recurrence $\left(\chi^{2}=7.58, \mathrm{df}=1\right.$, p $<0.01$ ). Male height was the second most important factor, and smoking cessation the only other variable which significantly influenced the risk of recurrence.

Conclusions - Analysis of several potential risk factors revealed that recurrence was not related to the BMI of the patient, the initial treatment of the pneumothorax, nor to its size. Recurrence was more common in taller men and in women. Smoking cessation appeared to reduce the risk of recurrence. These findings are discussed in the context of the possible aetiology of spontaneous pneumothorax, recurrences, and the management thereof.

(Thorax 1997;52:805-809)

Keywords: primary spontaneous pneumothorax, recurrence.

The pathophysiology of pneumothorax was described by Combulsier in 1747 . He recognised that the decrease in pulmonary function was a result of lung compression due to air in the pleural space. ${ }^{1}$ The term pneumothorax was first coined by Itard in $1803,{ }^{2}$ and the clinical features of this condition were described by Laennec in $1819 .^{2}$ Most cases of spontaneous pneumothorax were regarded as being sec- ondary to pulmonary tuberculosis up to 1932 when Kjaergard described primary spontaneous pneumothorax (PSP) as a separate entity occurring in previously healthy adults. ${ }^{3}$ Devilliers was the first to suggest the rupture of subpleural blebs as a cause for spontaneous pneumothorax and Hewson described thoracocentesis for its relief. ${ }^{1}$

PSP is a common clinical problem occurring in apparently healthy subjects with a reported incidence of 7.4-28/100 000 per year for men and 1.2/100 000 per year for women. ${ }^{4}$ The incidence of PSP is believed to be increasing, and the recurrence rate has been variably reported as $20-60 \%{ }^{5}$ with few studies of predisposing risk factors. ${ }^{67}$

This retrospective study, which preceded publication of the BTS guidelines, ${ }^{8}$ was conducted to evaluate the recurrence rate of PSP with particular reference to the following variables: age, sex, height and BMI of the patient, initial size of the pneumothorax, smoking status of the patient, and the primary form of treatment employed.

\section{Methods}

The study was carried out at a cardiothoracic surgical centre (Castle Hill Hospital) for the region of East Yorkshire. A total of 429 patients with a diagnosis of pneumothorax were identified from a computer-generated diagnostic index for the period from February 1990 to March 1994. A proportion of patients $(n=$ $104,24 \%$ ) were tertiary referrals for surgery from neighbouring hospitals and were excluded from the analysis to avoid selection bias. Missing data (case notes or radiographs) for 34 patients $(8 \%)$ left 291 case records for detailed study.

Patients were entered into the study if they were older than 15 years and if the initial index episode of pneumothorax occurred during the study period. The following data were collected: (1) age and sex, (2) height and weight, (3) smoking habits, (4) associated lung diseases, (5) side and size of pneumothorax, and (6) the treatment modalities. Patients with secondary, iatrogenic, and traumatic pneumothoraces were excluded. Although formal pulmonary function tests were not obtained on all patients, the diagnosis of obstructive airways disease (COPD/asthma) was excluded by the clinical history, physical examination and simple spirometric testing. The follow up period ranged from 42 to 62 months (mean 54 months), during which details of recurrences and changes in smoking habit were noted. At the end of the study the data were confirmed 
Table 1 Causes of secondary spontaneous pneumothorax

\begin{tabular}{lcc}
\hline Diagnosis & $\begin{array}{l}\text { Single } \\
\text { pneumothorax }\end{array}$ & $\begin{array}{l}\text { Recurrent } \\
\text { pneumothorax }\end{array}$ \\
\hline COPD & 9 & 15 \\
Asthma & 4 & 6 \\
Bronchogenic carcinoma & 3 & \\
Sarcoidosis & 1 & \\
Bronchiectasis & 1 & \\
Alveolar microlithiasis & 1 & 1 \\
Hard metal lung disease & 1 & 1 \\
Previous TB & & \\
Marfan's syndrome & 1 & 23 \\
Atypical TB & 21 & \\
Total & &
\end{tabular}

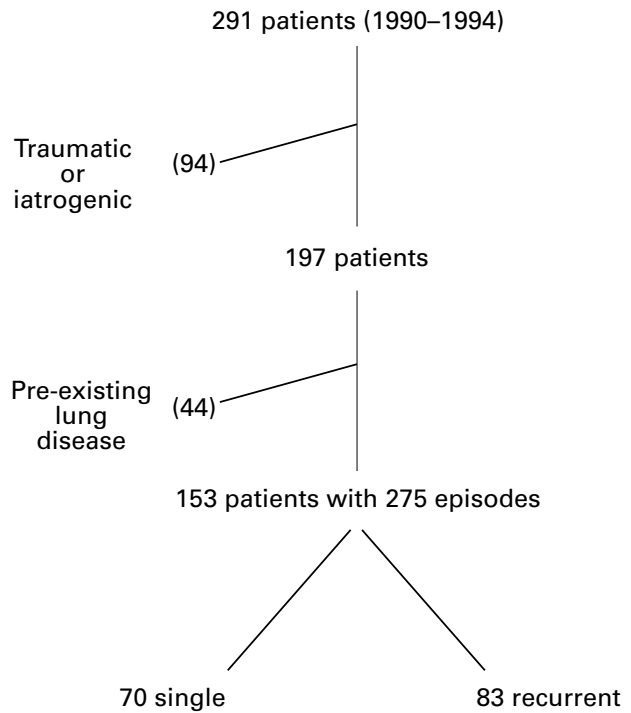

Figure 1 Patients excluded from the study (as shown in parentheses).

by a telephone interview with the patient or by a postal questionnaire.

The following definitions were employed: PSP was defined as pneumothorax occurring in a patient with no overt underlying lung disease, with no apparent alternative aetiology. Secondary pneumothorax was diagnosed by the concurrent presence of underlying lung disease such as asthma, COPD, lung cancer, etc. Recurrence was defined as a further pneumothorax occurring more than 30 days after the end of treatment in patients who had achieved full lung expansion following the initial pneumothorax. Size of pneumothorax was categorised according to the chest radiographic appearance as (a) shallow or small: a small rim of air remaining around the lung, (b) moderate: the lung being collapsed ap-

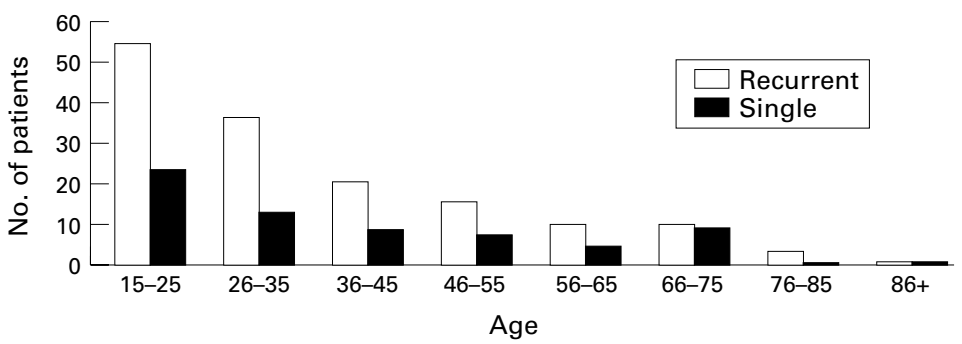

Figure 2 Age distribution. proximately half way towards the heart border, (c) airless lung separated from the diaphragm, (d) tension: pneumothorax associated with cardiorespiratory compromise.

Univariate analysis was carried out to determine whether each variable affected the risk of recurrence, using $\chi^{2}$ testing for categorical variables (sex, smoking status, smoking cessation, treatment type, and pneumothorax size) and $t$ tests for continuous measures (age, BMI, and height). BMI and height were analysed separately for men and women as these measures are not comparable between sexes. The combined effect of variables on the probability of recurrence was modelled using logistic regression. The effect of each variable on the number of recurrences was tested using $\chi^{2}$ tests for categorical variables and $t$ tests or $\mathrm{F}$ statistics for continuous variables.

\section{Results}

In the study period of four years 291 patients with a diagnosis of pneumothorax were reviewed. Patients with traumatic or iatrogenic pneumothorax $(\mathrm{n}=94)$, and those secondary to pre-existing lung disease $(\mathrm{n}=44$, table 1$)$ were excluded from the study (fig 1). Most of the patients with secondary pneumothorax had underlying obstructive airways disease (asthma or COPD, $\mathrm{n}=34,77 \%$ ).

Ages ranged from 15 to 95 with a mean (SD) of 36.8 (17.4) years and the age distribution was similar in both sexes. The peak incidence of PSP was in the first two decades of adult life (fig 2). Spontaneous pneumothorax was found to be twice as common in men as in women $(n=49,32 \%)$. The height of the patients ranged from 60 to 75 inches (mean (SD) 66.8 (3.6) inches). Two of the women correlated episodes of pneumothorax with their menstrual cycle and were therefore regarded as cases of catamenial pneumothorax.

One hundred and fifty three patients with PSP experienced 275 episodes, a single episode in 70 patients while 83 experienced recurrent episodes (fig 1). The overall recurrence rate was therefore $54 \%$. Sixty patients experienced one recurrence in the study period, $14 \mathrm{ex}-$ perienced two recurrences (12 patients experienced episodes outside the study period), and nine patients experienced more than two recurrences (three patients experiencing episodes outside the study period).

Seventy patients experienced left sided episodes only, 54 experienced right sided episodes only, and 24 experienced bilateral episodes at various points in time (one patient presenting with a bilateral pneumothorax). Twenty eight patients presented with a shallow pneumothorax, 74 with a moderate, and 46 with a large pneumothorax. A tension pneumothorax was recorded in five patients. This study preceded the publication of the BTS guidelines so that a standard management protocol did not exist, patients being treated at the discretion of the clinician involved. Nine patients were initially managed by aspiration, six requiring subsequent insertion of an intercostal chest drain 
Table 2 Primary risk factors in the recurrence of spontaneous pneumothorax (univariate analysis)

\begin{tabular}{|c|c|c|c|}
\hline Risk factor & $\begin{array}{l}\text { Recurrence } \\
n(\%)\end{array}$ & $p$ value $\left(\chi^{2}\right)$ & Significance \\
\hline \multicolumn{4}{|l|}{ Sex } \\
\hline Male & $48(46.2)$ & \multirow{2}{*}{0.0059} & \multirow{2}{*}{$* *$} \\
\hline Female & $35(71.4)$ & & \\
\hline $\begin{array}{l}\text { Smoking status } \\
\text { Yes }\end{array}$ & $64(55.7)$ & & \\
\hline & $19(50.0)$ & 0.676 & - \\
\hline $\begin{array}{l}\text { Smoking cessation } \\
\text { Yes }\end{array}$ & $6(40.0)$ & & \\
\hline $\begin{array}{l}\text { No } \\
\text { Treatment }\end{array}$ & $59(70.2)$ & 0.023 & * \\
\hline Observation & $6(42.9)$ & 0616 & \\
\hline $\begin{array}{l}\text { ICD and } A+I C D \\
\text { Initial size }\end{array}$ & $70(53.8)$ & 0.010 & \\
\hline $\begin{array}{l}\text { Initial size } \\
\text { S } \\
\mathrm{M} \\
\mathrm{C}\end{array}$ & $\begin{array}{ll}38 & (50.7) \\
28 & (60.9)\end{array}$ & 0.507 & - \\
\hline & Yes (mean) No & $p$ value ( $t$ test $)$ & \\
\hline \multicolumn{4}{|l|}{$\mathrm{BMI}$} \\
\hline Male & 37.0 & 0.436 & - \\
\hline $\begin{array}{l}\text { Female } \\
\text { Height (inches) }\end{array}$ & 36.0 & 0.529 & - \\
\hline $\begin{array}{l}\text { Height (inches) } \\
\text { Male }\end{array}$ & 69.3 & 0.008 & $* *$ \\
\hline Female & 62.9 & 0.925 & - \\
\hline
\end{tabular}

$\mathrm{ICD}=$ intercostal drain; $\mathrm{A}=$ aspiration; $\mathrm{S}=$ shallow; $\mathrm{M}=$ moderate $\mathrm{C}=$ complete.

Table 3 Pattern of recurrence

\begin{tabular}{|c|c|c|c|c|c|}
\hline & \multicolumn{3}{|c|}{ Time to recurrence } & \multicolumn{2}{|c|}{ Side of recurrence } \\
\hline & Year 1 & Year 2 & Thereafter & Ipsilateral & Contralateral \\
\hline Smokers & 38 & 15 & 11 & 54 & 10 \\
\hline Non-smokers & 2 & 6 & 11 & 6 & 13 \\
\hline
\end{tabular}

(ICD) because of failed resolution of the pneumothorax. The initial management of 14 patients was simple observation. An ICD was inserted at the outset without prior aspiration in 124 patients and their recurrence rate $(53.8 \%$, table 2) closely resembled that for the entire group $(54.2 \%)$.

\section{INDIVIDUAL FACTORS INFLUENCING} RECURRENCE

An analysis of the effect on the risk of recurrence of various patient characteristics found that women were significantly more likely than men to develop a recurrence (corrected $\chi^{2}=7.58, \mathrm{df}=1, \mathrm{p}=0.006$; table 2 ).

Height and BMI were tested separately for men and women as the measures are not directly comparable between sexes. BMI for both sexes and the height of female patients were not shown to have a significant influence on recurrence, but the mean height of male patients with a recurrence was significantly higher than those without a recurrence $(t=$ $2.71, \mathrm{df}=80, \mathrm{p}=0.008$ ).

One hundred and fifteen (75\%) of patients were smokers on entry to the trial (characteristic $=$ "smoking status"). Whether a person was a smoker or not had no significant influence on subsequent recurrence. Data on changes in smoking habit (characteristic= "smoking cessation") were only available for 99 of the 115 patients. Only $10 \%$ of patients
Table 4 Logistic regression model for recurrence

\begin{tabular}{llll}
\hline Variable & $\begin{array}{l}\text { Regression } \\
\text { coefficient }(B)\end{array}$ & Significance & $\begin{array}{l}\text { Odds ratio } \\
\text { exp (B) }\end{array}$ \\
\hline Constant & -6.786 & 0.296 & \\
Sex & -8.728 & 0.179 & 0.0002 \\
Height & 0.103 & 0.313 & 1.108 \\
Sex $\times$ Height & 0.120 & 0.236 & 1.128 \\
\hline
\end{tabular}

stopped smoking after their first pneumothorax, but this group had a significantly lower recurrence rate $\left(40 \% ; \chi^{2}=5.16, \mathrm{df}=1, \mathrm{p}=\right.$ $0.023)$ than those patients who continued to smoke $(70 \%)$.

Other factors that did not significantly affect the recurrence rate were age, initial size of pneumothorax, and mode of management. However, a reliable assessment of the impact of the different forms of treatment employed cannot be made because of the small numbers in the treatment groups (aspiration group and aspiration/ICD group). Testing was only possible between the "observation alone" and ICD groups where no significant difference was shown.

An analysis of tabulations of recurrence by time and side of recurrence revealed no patterns for any of the study variables except smoking status. Smokers tended to develop recurrences earlier than non-smokers and on the same side as the initial pneumothorax (table 3). Since the numbers of non-smokers were low, and since this was a subdivision of the main outcome variable, no firm conclusions could be drawn from this.

LOGISTIC REGRESSION MODEL FOR RECURRENCE A logistic regression model was fitted for the logit (probability of a recurrence) with sex, BMI, smoking status, height, age, and size of pneumothorax considered as possible explanatory variables. Because of physiological differences, interactions between sex and each of the other variables were also considered as possible explanatory variables. Forward stepwise selection included the variables "sex" and "sex $\times$ height" in the model. With the forced addition of the "height" variable which reduced the significance of the selected variables the final model was as displayed in table 4 .

This model correctly predicted recurrence status in $65.8 \%$ of cases. The discriminatory power of the model is not a great improvement upon chance (which would predict $50 \%$ of cases) and is not therefore sufficient to predict accurately recurrence for individuals.

Interpretation of the parameter values is not straightforward because of the inclusion of the "sex $\times$ height" interaction. However, the results are in keeping with the findings of the univariate analysis that women have a greater risk of recurrence than men, with increased height in men making a recurrence more likely. No other variable or interaction contributed significantly to the model. In particular, smoking status and possible interactions between sex and smoking do not merit inclusion. 
Table 5 Primary risk factors in multiple recurrence of spontaneous pneumothorax (univariate analysis)

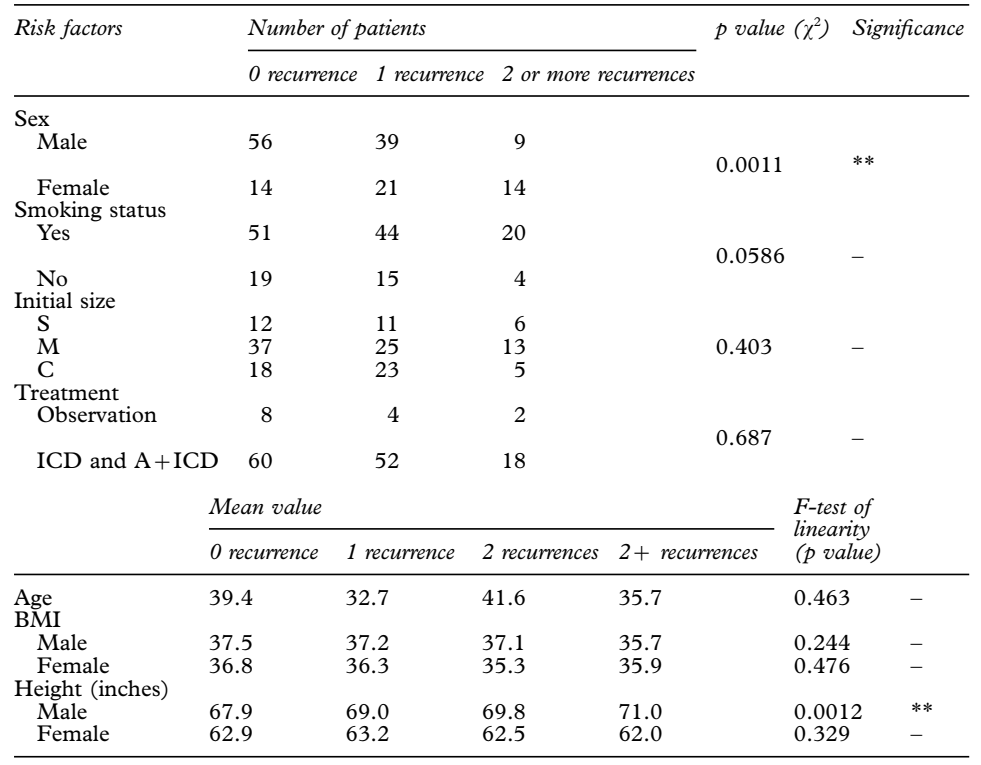

$\mathrm{ICD}=$ intercostal drain; $\mathrm{A}=$ aspiration; $\mathrm{S}=$ shallow; $\mathrm{M}=$ moderate $\mathrm{C}=$ complete.

INDIVIDUAL FACTORS INFLUENCING MULTIPLE RECURRENCES

When account was taken of the number of recurrences and the same variables tested (excluding "smoking cessation" for which data were not available), then the same two variables (sex and male height) were found to affect recurrence significantly (table 5). No new significant effects were found in comparison with the simple risk of recurrence analysis.

\section{Discussion}

This study confirms some of the previously established facts about the epidemiology of PSP. The male predominance found in our study, with a ratio of $2: 1$, may suggest a rising incidence of PSP in women. A similar observation was made by Primrose who also found a male to female ratio of $2: 1,{ }^{9}$ in comparison with previous studies which have shown a ratio of $6: 1-8: 1{ }^{410}$ The monophasic age distribution observed in this study, with a peak incidence in the first two decades of adult life, is due to the fact that the study was strictly confined to patients with PSP. Studies which have included cases of secondary spontaneous pneumothorax have demonstrated a biphasic age distribution with a second peak in late adult life, mainly due to underlying chronic obstructive airways disease. ${ }^{911}$ It is well established that smoking increases the risk of contracting a first pneumothorax, ${ }^{12}$ and our study supports this fact with $75.2 \%$ of our patients being smokers.

The main purpose of this study was to evaluate the factors predisposing to the recurrence of PSP, since few data on this are available. Surprisingly, although women are at a lower risk of developing the initial pneumothorax, their risk of recurrence is higher than men. What is the cause of this paradox? Perhaps the factors that influence the recurrence rate of PSP are different from those that cause PSP in the first instance, and these factors may be more influential in women than in men. Certain types of pneumothorax - for example, catamenial and those secondary to lymphangiolyomyomatosis - are specific to women. However, in our study population there were only two women with apparent catamenial pneumothoraces.

It is tempting to speculate that a hormonal factor might be operating to explain this difference between the sexes. Progesterone has been shown to have an effect on collagen. Administration of progesterone has been shown to inhibit the progression of emphysema in rats by inhibiting the activity of proteolytic enzymes such as elastase and collagenase, similar to that of $\alpha$-antitrypsin and pulmonary surface active lipoproteins. ${ }^{13}$ In men the development of idiopathic pneumothorax has been shown to be associated with low progesterone levels due to hypofunction of the adrenal cortex. ${ }^{14}$ It is possible that low progesterone levels, induced by cyclical hormonal variation, may predispose women to recurrence of PSP.

Smoking is clearly implicated in the development of the initial pneumothorax. Most of our patients were smokers, and smoking cessation seemed to reduce the recurrence rate. The pattern of recurrence in smokers seemed to differ from that seen in non-smokers, with recurrence tending to occur earlier and on the same side as the initial pneumothorax. This risk might simply be mediated by an increased tendency to coughing and transmission of increased intrathoracic pressures to an area of healing visceral pleura, or it may be associated with structural changes consequent upon the reduced antiprotease activity that is associated with the smoking habit.

The risk of developing a spontaneous pneumothorax or a recurrence is probably multifactorial. Our multivariate analysis indicated that the height of male patients was linked to the risk of recurrence; although this factor was not evident in our female patients, they, of course, were a cohort of smaller average height. Bullae and blebs are commonly found at the apex of lungs of patients with spontaneous pneumothorax. It has been suggested that these are formed as a result of degradation of elastic fibres due to a focal imbalance between elastase and $\alpha_{1}$-antitrypsin. ${ }^{15}$ They may also result, and be more likely to rupture, from the increased forces generated at the apex of the lungs of tall subjects. ${ }^{16}$

Further factors may also operate. A study by Bense $e t a l^{17}$ on a group of patients with PSP who had never smoked and had a normal $\alpha_{1-}$ antitrypsin level showed bilateral bronchial abnormalities in most patients at bronchoscopic examination. A case of recurrent PSP has also been reported in a non-smoker with bronchial atresia. ${ }^{18}$ It is possible that the presence of such anatomical abnormalities, not visualised on plain chest radiographs, may be responsible for the causation and bilateral recurrences of pneumothorax.

The initial management of pneumothorax remained controversial until the publication of BTS guidelines in 1994. Our study supports 
the fact that the insertion of an intercostal drain does not prevent recurrence. ${ }^{19}$ As yet, there is no uniform consensus regarding the management of recurrent episodes and the approach is fairly individual. Some clinicians, influenced by the approximate $50 \%$ recurrence rate, advocate an aggressive approach with early operative pleurodesis, ${ }^{20}$ whilst the remainder are more conservative. Our findings might influence the management of primary spontaneous pneumothorax. All such patients should be strongly encouraged to stop smoking since this could almost halve the recurrence rate. The enhanced risk of recurrence on the same side in those who did not stop smoking might be a factor in the consideration of an early thoracoscopic procedure in such patients, especially in those who are exceptionally tall.

In conclusion, we confirm the high incidence of recurrence of spontaneous pneumothorax. Whereas this is not related to the build of the patient, as judged by the BMI, or to the type of non-surgical treatment of the pneumothorax, it is influenced by the sex and height of the patient, and by smoking cessation. We believe the aetiology of PSP to be multifactorial and suspect that the interrelationship of these factors is complex, requiring further studies to provide a better understanding.

The authors wish to thank Dr M A Greenstone and Dr D V McGivern for permission to study their patients, and John Arnold for his help in preparation of the manuscript.

1 Withers JN, Fishback ME, Kiehl PV, Hannon JL. Spontaneous pneumothorax: suggested aetiology and comparison of treatment methods. Am f Surg 1964;108:772-6.
2 Laennec RTH. Traite de l'auscultation mediate et des maladies des poumons et du coeur. Tome Second. Paris, 1819.

3 Kjaergard $\mathrm{H}$. Spontaneous pneumothorax in the apparently healthy. Acta Med Scand (Suppl) 1932;43:1-159.

4 Melton LJ, Hepper NGG, Offord KP. Incidence of spontaneous pneumothorax in Olmsted County, Minnesota: 1950-1974. Am Rev Respir Dis 1979;120:1379-82.

5 Parry GW, Juniper ME, Dussek JE. Surgical intervention in spontaneous pneumothorax. Respir Med 1992;86:1-2.

6 Lippert HL, Lund O, Blegvad S, Larsen HV. Independent risk factors for cumulative recurrence rate after first spon-

Sadikot RT, Meadow tano taneous pneumothorax

8 Miller AC, Harvey JE. Guidelines for the management of spontaneous pneumothorax. BMF 1993;307:114-6.

9 Primrose WR. Spontaneous pneumothorax: a retrospective review of aetiology, pathogenesis and management. Scott review of aetiology, pa

10 Neal JF, Vargas G, Smith D, Aki BF, Edwards WS. Bilateral bleb excision through median sternotomy. Am $\mathcal{F}$ Surg 1979;138:794-7.

11 Ruckley CV, McCormack RJM. The management of spontaneous pneumothorax. Thorax 1966;21:139-44.

12 Bense L, Eklund G, Wiman LG. Smoking and increased risk of contracting spontaneous pneumothorax. Chest 1987;92 1009-12.

13 Ito H, Aviado DM. Prevention of pulmonary emphysema in rats by progesterone. 7 Pharmacol Exp Ther 1968;161: 197-204

14 Nakamura H, Konishiike J, Sugamara A, Takeno Y. Epidemiology of spontaneous pneumothorax in women. Chest 1986;89:378-82.

15 Fukuda Y, Haraguchi S, Tanaka S, Yamanaka N. Pathogenesis of blebs and bullae of patients with spontaneous pneumothorax. Ultrastructural and immunohistochemical studies. Am 7 Respir Crit Care Med 1994;149:A1022.

16 west JB. Distribution of mechanical stress in the lung, a possible factor in localisation of pulmonary disease. Lancet possible factor

17 Bense L, Eklund G, Wiman LG. Bilateral bronchial anomaly: a pathogenetic factor in spontaneous pneumothorax. Am Rev Respir Dis 1992;146:513-6.

18 Berkman N, Bar-Ziv J, Breuer R. Recurrent spontaneous pneumothorax associated with bronchial atresia. Respir Med 1996;90:307-9.

19 Harvey J, Prescott RJ on behalf of British Thoracic Society Research Committee. Simple aspiration versus intercostal tube drainage for spontaneous pneumothorax in patient with normal lungs. BMF 1994;309:1338-9.

20 Light RW. Management of spontaneous pneumothorax. Am 